\title{
What Can Lipids in Anti-neutrophil Cytoplasmic Antibody-associated Vasculitis Tell Us?
}

\author{
Chan-Bum Choi, M.D., Ph.D., MPH \\ Department of Rheumatology, Hanyang University Hospital for Rheumatic Diseases, Seoul, Korea
}

Anti-neutrophil cytoplasmic antibody (ANCA)-associated vasculitis (AAV) are systemic inflammatory autoimmune diseases with diverse clinical manifestations mainly involving small-sized blood vessels. Our understanding of AAV have significantly improved since the first description of a patient now suspected of granulomatosis with polyangiitis in 1931 by Klinger [1]. Association with ANCA was first reported by van der Woude et al. [2] in 1985. While its value of ANCA in diagnosis of AAV is well accepted [3], its association with disease activity is controversial [4].

The 1990 American College of Rheumatology classification criteria, 2007 European Medicine Agency algorithm for the classification of AAV, and revised 2012 Chapel Hill consensus conference nomenclature of vasculitides aids us in the diagnosis of AAV. Clinical features as well as imaging and laboratory tests and most importantly histology make up the diagnosis. But besides the clinical manifestations, other aspects of the disease have very limited impact on assessment of disease activity. The international Outcome Measures in Rheumatology (OMERACT) Vasculitis Working Group have endorsed the Birmingham Vasculitis Activity Score (BVAS) for assessment of disease activity in AAV [5]. Erythrocyte sedimentation rate (ESR), C-reactive protein (CRP), ANCA, B-lymphocytes, and gamma-globulins has been used in randomized clinical trials to assess disease activity, but their validity remains unclear [6].

Efforts to identify biomarkers for AAV have suggested neutrophil microparticle, neutrophil extracellular traps, urinary MCP-1, urinary soluble CD16, CXCL13, matrix metalloproteinase-3, tissue inhibitor of metalloproteinases-1, S100A8/A9, mobilitygroupbox-1 protein, typical damage-associated molecular pattern protein, C3a, $\mathrm{C} 5 \mathrm{a}$, and soluble C5b-9 as potential candidates [7]. Other possible candidate includes neutrophil to lymphocyte ratio [8], platelet to lymphocyte ratio [9], hepcidin [10], IL-6 [11], d-dimer [12], vitamin D levels [13], soluble lectin-like oxidized low-density lipoprotein receptor 1 [14], mannose-binding lectin levels [15], and lipid levels [16]. But there is no validated biomarker for assessment of disease activity in AAV.

The outcomes of AAV have greatly improved with the use of glucocorticoids and cyclophosphamides and now with rituximab. But it can show frequent relapses and adverse events from therapies especially with glucocorticoids can cause significant morbidities. Comorbidities are also an important aspect of management of AAV. Increased cardiovascular risk in patients with $\mathrm{AAV}$ is well recognized warranting active management [17].

Wallace et al. [16] showed temporal relationship between change in lipid levels and remission induction in a post hoc analysis of the Rituximab for ANCA-Associated Vasculitis (RAVE) trial. They concluded that the assessment for cardiovascular risk using lipid profiles in the initial phase after the diagnosis of AAV before remission induction may not accurately reflect the true risk. Patients with AAV are at increased risk for cardiovascular disease and timing of lipid profile assessment may have a role in its active management. It also implies that lipid profile decreases in active disease state with active inflammation which is consistent with findings in other inflammatory

Received : December 28, 2020, Accepted : December 28, 2020

Corresponding to : Chan-Bum Choi iD http://orcid.org/0000-0002-4691-5455

Department of Rheumatology, Hanyang University Hospital for Rheumatic Diseases, 222-1 Wangsimni-ro, Seongdong-gu, Seoul 04763, Korea. E-mail : cbchoi@hanyang.ac.kr

Copyright (c) 2021 by The Korean College of Rheumatology.

This is an Open Access article, which permits unrestricted non-commerical use, distribution, and reproduction in any medium, provided the original work is properly cited. 
rheumatic diseases.

Ahn et al. [18] also demonstrated relationship between lipid profile and disease activity in AAV in a cross-sectional study. It was irrespective of treatment of corticosteroids and immunosuppressant and apolipoprotein A1 (apoA1) showed the most significant correlation. ApoA1 is a known acute phase reactant and its correlation with acute coronary syndrome in high risk population have been demonstrated [19]. Apolipoprotein B (apoB) to apoA1 ratio has been shown to correlate with cardiovascular risk [20].

Multiple studies show correlation between lipid profiles and disease activity in AAV. The phenomenon may not be specific to AAV, but rather a response to persistent active inflammation that can be seen in autoimmune inflammatory diseases. Similar results has been shown in other inflammatory rheumatic diseases. Decrease levels of total cholesterol, low-density lipoprotein (LDL), and high-density lipoprotein (HDL) with active rheumatoid arthritis (RA) and their increase after decrease in RA disease activity with treatment have been observed correlating with cardiovascular risk, hence the lipid paradox [21]. The correlation between lipid profiles and CRP has also been observed [22]. Nevertheless, we are lacking valid biomarkers of disease activity in AAV and it would be valuable if lipid profiles, which are routinely assessed, can provide information on AAV disease activity.

Management of cardiovascular risk is an important part of AAV management. Lifestyle modification including smoking cessation and management of blood pressure and dyslipidemia is recommended. Assessment of dyslipidemia is an important part of cardiovascular risk assessment and it has been shown that the results may be altered with AAV disease activity. Using lipid profile in active stage of AAV disease activity may underestimate cardiovascular risk and undermine efforts for active management.

Non-HDL cholesterol based assessment are generally accepted for cardiovascular risk stratification in general population. However, dysfunctional proinflammatory HDL has been suggested as a key component of increased cardiovascular risk in autoimmune inflammatory diseases such as systemic lupus erythematosus (SLE) [23]. Ahn et al. [18] have shown that apoA1, an important component of HDL, is significantly associated with AAV disease activity and it will be interesting to see how it correlates with cardiovascular diseases.

Lipid profile should be routinely assessed in patients with AAV and it can provide valuable information. Evidence show that avoiding the use of lipid profile during active inflammatory phase of AAV is advisable when interpreting the results for cardiovascular risk assessment. It may in turn suggest that lipid profile can add information in assessing disease activity in AAV.

\section{CONFLICT OF INTEREST}

No potential conflict of interest relevant to this article was reported.

\section{REFERENCES}

1. Klinger $\mathrm{H}$. [Grenzformen der periarteritis nodosa]. Frankf $\mathrm{Z}$ Pathol 1931;42:455-80. German.

2. van der Woude FJ, Rasmussen N, Lobatto S, Wiik A, Permin $\mathrm{H}$, van Es LA, et al. Autoantibodies against neutrophils and monocytes: tool for diagnosis and marker of disease activity in Wegener's granulomatosis. Lancet 1985;1:425-9.

3. Bossuyt X, Cohen Tervaert JW, Arimura Y, Blockmans D, Flores-Suárez LF, Guillevin L, et al. Position paper: revised 2017 international consensus on testing of ANCAs in granulomatosis with polyangiitis and microscopic polyangiitis. Nat Rev Rheumatol 2017;13:683-92.

4. Osman MS, Tervaert JWC. Anti-neutrophil cytoplasmic antibodies (ANCA) as disease activity biomarkers in a "personalized medicine approach" in ANCA-associated vasculitis. Curr Rheumatol Rep 2019;21:76.

5. Merkel PA, Aydin SZ, Boers M, Direskeneli H, Herlyn K, Seo $\mathrm{P}$, et al. The OMERACT core set of outcome measures for use in clinical trials of ANCA-associated vasculitis. J Rheumatol 2011;38:1480-6.

6. Monti S, Quinn KA, Christensen R, Jayne D, Langford C, Lanier GE, et al. Use and reporting of outcome measures in randomized trials for anti-neutrophil cytoplasmic antibody-associated vasculitis: a systematic literature review. Semin Arthritis Rheum 2020;50:1314-25.

7. Csernok E, Hellmich B. Usefulness of vasculitis biomarkers in the era of the personalized medicine. Autoimmun Rev 2020;19:102514.

8. Ahn SS, Jung SM, Song JJ, Park YB, Lee SW. Neutrophil to lymphocyte ratio at diagnosis can estimate vasculitis activity and poor prognosis in patients with ANCA-associated vasculitis: a retrospective study. BMC Nephrol 2018;19: 187.

9. Park HJ, Jung SM, Song JJ, Park YB, Lee SW. Platelet to lymphocyte ratio is associated with the current activity of ANCA-associated vasculitis at diagnosis: a retrospective monocentric study. Rheumatol Int 2018;38:1865-71.

10. Přikryl $P$, Hrušková Z, Konopásek $P$, Hladinová Z, Tesař V, Vokurka M. Serum hepcidin is increased in ANCA-associated vasculitis and correlates with activity markers. Physiol Res 2018;67:945-54.

11. Berti A, Warner R, Johnson K, Cornec D, Schroeder DR, Kabat BF, et al.; RAVE-ITN Research Group. The association of serum interleukin- 6 levels with clinical outcomes in 
antineutrophil cytoplasmic antibody-associated vasculitis. J Autoimmun 2019;105:102302.

12. Borowiec A, Dąbrowski R, Kowalik I, Rusinowicz T, Hadzik-Błaszczyk M, Krupa R, et al. Elevated levels of d-dimer are associated with inflammation and disease activity rather than risk of venous thromboembolism in patients with granulomatosis with polyangiitis in long term observation. Adv Med Sci 2020;65:97-101.

13. Yoon T, Ahn SS, Pyo JY, Song JJ, Park YB, Lee SW. Serum vitamin D level correlates with disease activity and health-related quality of life in antineutrophil cytoplasmic antibody-associated vasculitis. Z Rheumatol 2020 Dec 18 [Epub]. DOI: 10.1007/s00393-020-00949-2.

14. Yoon T, Ahn SS, Song JJ, Park YB, Lee SW. Soluble lectin-like oxidized low-density lipoprotein receptor 1 is inversely correlated with the activity of ANCA-associated vasculitis. Yonsei Med J 2020;61:720-5.

15. Yoon T, Ahn SS, Yoo J, Yoo BW, Kwon HC, Jung SM, et al. Serum Mannose-binding lectin levels are correlated with the disease activity of antineutrophil cytoplasmic antibody-associated vasculitis: a single-center study. Tohoku J Exp Med 2020;251:117-23.

16. Wallace ZS, Fu X, Liao K, Kallenberg CGM, Langford CA, Merkel PA, et al. Disease activity, antineutrophil cytoplasmic antibody type, and lipid levels in antineutrophil cytoplasmic antibody-associated vasculitis. Arthritis Rheumatol 2019;71:1879-87.

17. Houben E, Penne EL, Voskuyl AE, van der Heijden JW, Otten RHJ, Boers M, et al. Cardiovascular events in an- ti-neutrophil cytoplasmic antibody-associated vasculitis: a meta-analysis of observational studies. Rheumatology (Oxford) 2018;57:555-62.

18. Ahn SS, Yoon T, Song JJ, Park YB, Lee SW. Lipid profiles in anti-neutrophil cytoplasmic antibody-associated vasculitis: a cross-sectional analysis. J Rheum Dis 2020;27:261-9.

19. Soria-Florido MT, Castañer O, Lassale C, Estruch R, Salas-Salvadó J, Martínez-González MÁ, et al. Dysfunctional high-density lipoproteins are associated with a greater incidence of acute coronary syndrome in a population at high cardiovascular risk: a nested case-control study. Circulation 2020;141:444-53.

20. Walldius G, Jungner I, Holme I, Aastveit AH, Kolar W, Steiner E. High apolipoprotein B, low apolipoprotein A-I, and improvement in the prediction of fatal myocardial infarction (AMORIS study): a prospective study. Lancet 2001; 358:2026-33.

21. Robertson J, Peters MJ, McInnes IB, Sattar N. Changes in lipid levels with inflammation and therapy in RA: a maturing paradigm. Nat Rev Rheumatol 2013;9:513-23.

22. Ridker PM, Danielson E, Fonseca FA, Genest J, Gotto AMJr, Kastelein JJ, et al.; JUPITER Trial Study Group. Reduction in C-reactive protein and LDL cholesterol and cardiovascular event rates after initiation of rosuvastatin: a prospective study of the JUPITER trial. Lancet 2009;373:1175-82.

23. Kim SY, Yu M, Morin EE, Kang J, Kaplan MJ, Schwendeman A. High-density lipoprotein in lupus: disease biomarkers and potential therapeutic strategy. Arthritis Rheumatol 2020;72:20-30 